\title{
ON A UNIQUE SOLUTION OF A DIFFERENTIAL EQUATION IN VECTOR DISTRIBUTION
}

\author{
Submitted for publication to \\ Mathematical Notes (Matematicheskiye Zametki) \\ Moscow, Russia \\ MICHAEL V. BASIN \\ Institute of Control Science, Moscow, Russia \\ 36-1-135, Matveevskaya ul., Moscow 119517, Russia \\ E-mail:postavl@icsan.msk.su
}

Let us consider a differential equation in vector distribution

$$
\dot{x}(t)=f(x, u, t)+b(x, u, t) \dot{u}(t), \quad x\left(t_{0}\right)=x_{0},
$$

where $x \in R^{n}$, functions $f(x, u, t) \in R^{n}$ and $b(x, u, t) \in R^{n \times m}$ are piecewise continuous in $x, u, t$, and $u(t)=\left(u_{1}(t), \ldots, u_{m}(t)\right) \in R^{m}$ is a bounded variation function which is non-decreasing in the following sense: $u\left(t_{2}\right) \geq u\left(t_{1}\right)$ as $t_{2} \geq t_{1}$, if $u_{i}\left(t_{2}\right) \geq u_{i}\left(t_{1}\right)$ for all $i=1, \ldots, m$.

A solution to equation (1) is defined as a vibrosolution [1], i.e., as a unique limit of absolutely continuous solutions to pre-limiting equations (1) corresponding to absolutely continuous nondecreasing approximations of function $u(t)$. Solutions to pre-limiting equations (1) are regarded as conventional solutions of ordinary differential equations with discontinuous right-hand sides in the sense of Filippov [2]. A vibrosolution is expected to be a function discontinuous at discontinuity points of the function $u(t)$.

Theorem 1: Let the following conditions hold:

(1) functions $f(x, u, t), b(x, u, t) \partial b(x, u, t) / \partial x, \partial b(x, u, t)) / \partial t$ are piecewise continuous domains are locally connected;

(2) functions $f(x, u, t), b(x, u, t)$ satisfy the one-side Lipschitz condition in $x$ [2].

A unique vibrosolution to equation (1) exists if and only if an $n \times m$-dimensional system of differential equations in differentials

$$
\frac{d \xi}{d u}=b(\xi, u, x), \quad \xi(\omega)=z,
$$

is solvable inside a cone $K=\left\{u \in R^{m}: u_{i} \geq \omega_{i}, i=1, \ldots, m\right\}$ with arbitrary initial values $\omega \in R^{m}$, $\omega \geq u\left(t_{0}\right), z \in R^{n}$, and $s \geq t_{0}$.

Since a vibrosolution is a discontinuous solution to equation (1), it is helpful to design an equation with a measure which enables us to compute jumps of a vibrosolution at discontinuity points of function $u(t)$. This completely determines the behavior of a vibrosolution.

Theorem 2: Let the conditions of Theorem 1 hold. Then a solution of an equivalent equation with a measure

$$
d x(t)=f(x, u, t) d t+b(x, u, t) d u^{c}(t)+\sum_{t_{i}} G\left(x\left(t_{i}-\right), u\left(t_{i}-\right), \Delta u\left(t_{i}\right), t_{i}\right) d \chi\left(t-t_{i}\right), x\left(t_{0}\right)=x_{0}
$$

coincides with a vibrosolution of equation (1), if $G(z, v, u, s)=\xi(z, v, v+u, s)-z$, where 
$\xi(z, v, u, s)$ is a solution to system $(2) ; u^{c}(t)$ is a continuous component of a nondecreasing function $u(t), \Delta u\left(t_{i}\right)=u\left(t_{i}+\right)-u\left(t_{i}-\right)$ is a jump of a function $u(t)$ at $t_{i}, t_{i}$ are discontinuity points of a function $u(t)$, and $\chi\left(t-t_{i}\right)$ is the Heaviside function.

\section{References}

[1] Krasnosel'skii, M.A., Pokrovskiy, A.V., Systems with Hysteresis, Springer-Verlag, Berlin 1989.

[2] Filippov, A.F., Differential Equations with Discontinuous Right-Hand Sides, Kluwer, New York 1988. 


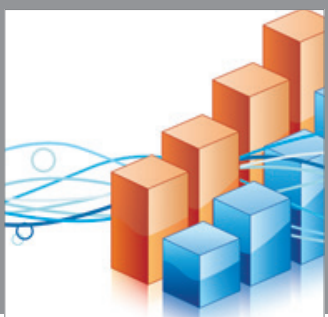

Advances in

Operations Research

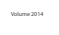

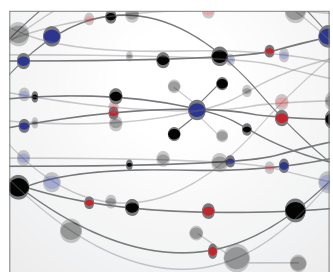

\section{The Scientific} World Journal
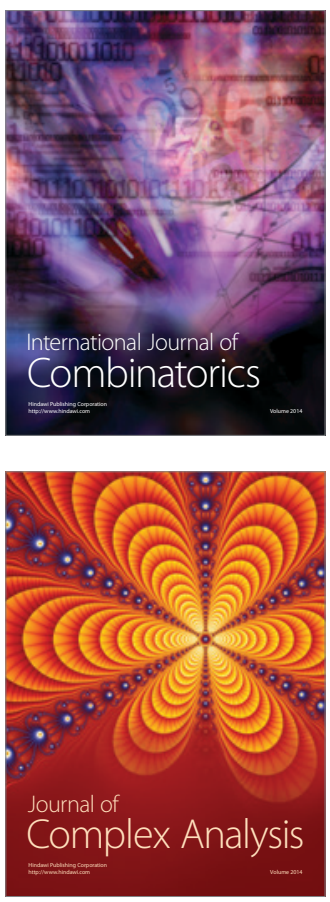

International Journal of

Mathematics and

Mathematical

Sciences
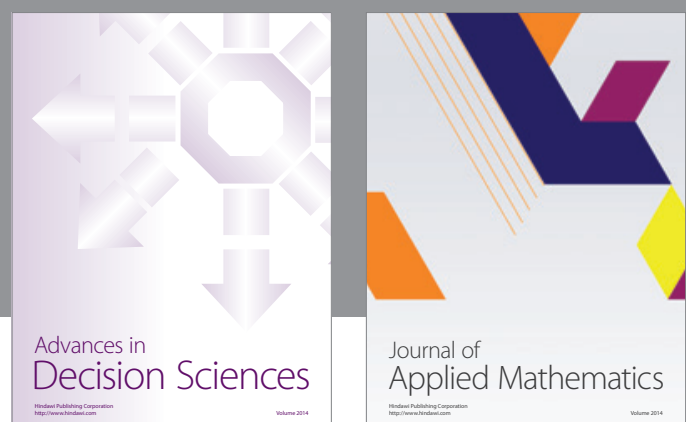

Journal of

Applied Mathematics
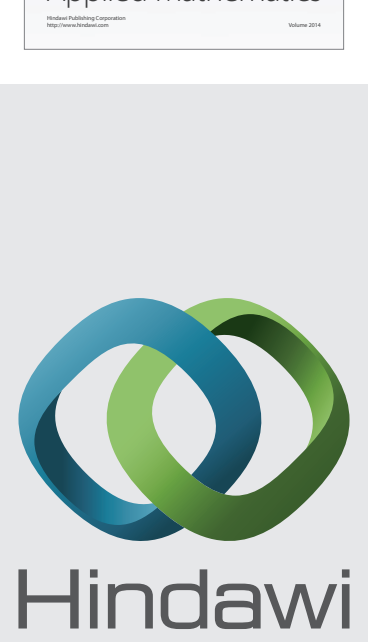

Submit your manuscripts at http://www.hindawi.com
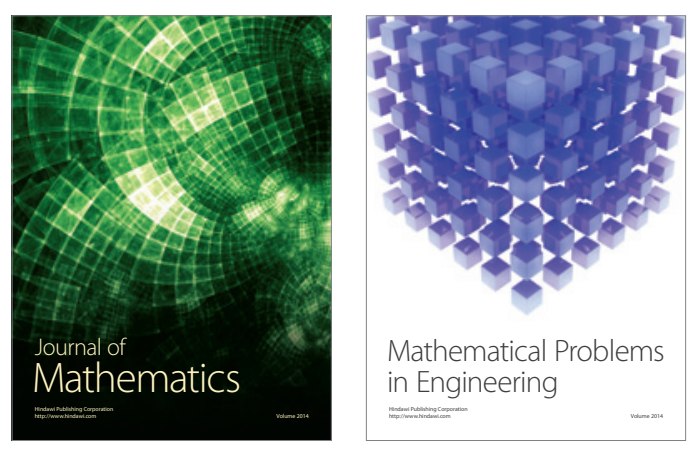

Mathematical Problems in Engineering
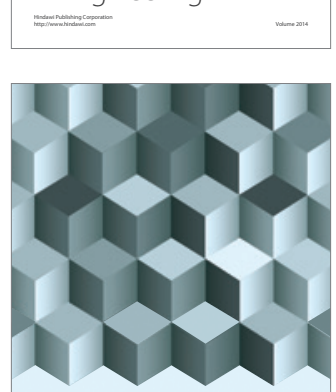

Journal of

Function Spaces
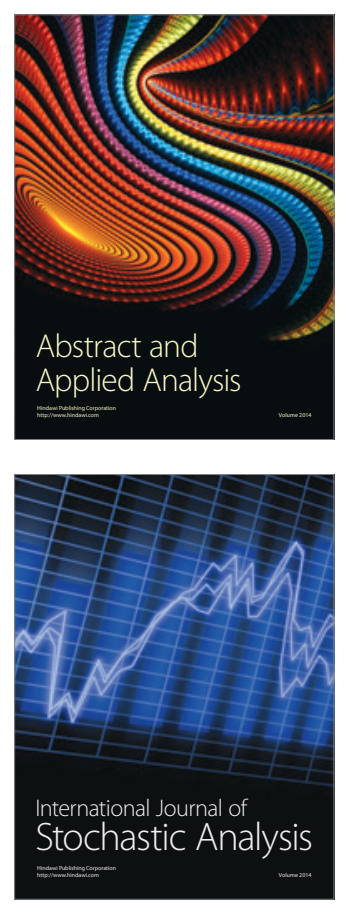

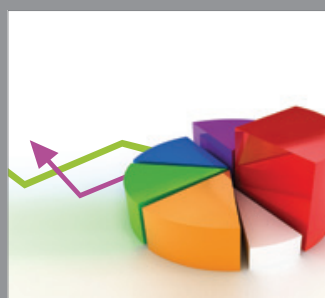

ournal of

Probability and Statistics

Promensencen
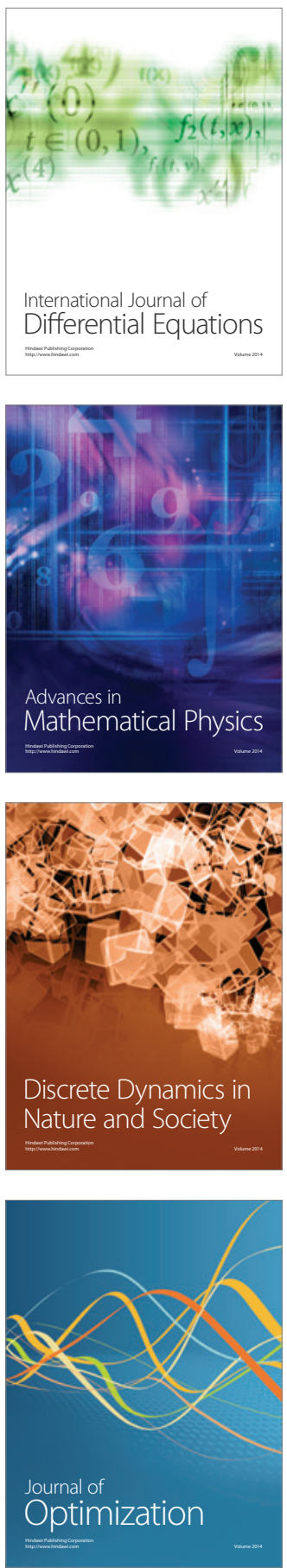